\title{
Long-term stability and sustainability evaluation for mode-locked fiber laser with graphene/PMMA saturable absorbers
}

\begin{abstract}
A quality saturable absorber for the integration of a practical laser product with long-term stability and sustainability is of utmost importance to be delivered to end-users. Graphene/polymethyl-methacrylate saturable absorber is proposed with the advantage of gapless material characteristic for ultra-broadband wavelength operation. This saturable absorber possesses stability over 24-hour continuous mode-locked laser operation at $105.2 \mathrm{~mW}$ pump power, as well as sustainability over four consecutive observation weeks with the preservation of $670 \mathrm{fs}$ pulse duration within the experimental period. Last but not least, the proposed saturable absorber shows excellent long-term stability and sustainability for the formulation of practical seed mode-locked fiber laser source.
\end{abstract}

Keyword: Saturable absorber; Mode-locking; Fiber laser; Stability; Sustainability 